\title{
Management of Retropharyngeal Node Metastases from Thyroid Carcinoma: Reply
}

\author{
Dana M. Hartl ${ }^{1}$
}

Published online: 6 October 2015

(c) Société Internationale de Chirurgie 2015

Thank you for your interest in our work and for your comments and questions. Concerning an eventual correlation between the tumor location in the thyroid lobe (superior pole, middle third, or lower third) and the incidence of retropharyngeal nodes, there is not enough data in the literature to make any meaningful conclusions. In our small series, one patient (patient number 3 ) did indeed have a tumor in the upper pole of the left lobe (as well as two tumor foci in the right lobe), with only one level II lymph node metastasis on the left side (without metastases in levels III or IV) in addition to the left retropharyngeal node. Patient one had a middle-third tumor on the side of the retropharyngeal node, but also two smaller tumors in the contralateral lobe. Patient number 5 had a diffuse sclerosing papillary carcinoma with multiple diffuse tumor foci throughout the thyroid. Our other two patients had initial surgery outside of our institution and we do not have data regarding the tumor location or the exact location of the initial lymph node metastases. What is interesting to note, however, is that all of our patients initially had macroscopic lateral lymph node metastases (N1b) and the three patients initially treated at our institution all had metastatic nodes in level II ipsilateral to the retropharyngeal node. This lends to the theory of retropharyngeal node metastases via the lateral jugular chain, but with three cases, no strong conclusions can be made. In each surgical case, only one metastatic node was found in each retropharyngeal space, with no other nodes seen during surgery or resected in the specimen.

Dana M. Hartl

dana.hartl@gustaveroussy.fr

1 Department of Head and Neck Oncology, Institut de Cancérologie Gustave Roussy, Villejuif, France 